\title{
Conception de sites web adaptée à l'âge
}

\author{
Alexander Seifert, Alireza Darvishy \& Stephan Roth
}

Les sites Web doivent « être adaptés à l'âge et utilisables par tous ». Cette exigence a été explorée dans un projet de recherche interdisciplinaire qui a débouché sur une brochure utile pour la pratique.

\section{La recherche menée auprès des seniors donne lieu}

\section{à des recommandations pratiques}

En plus du manque d'assistance personnelle, ce sont principalement des obstacles et des peurs personnels, selon l'étude Seifert et Schelling (voir article dans cette revue), qui compliquent l'utilisation d'Internet. A côté de cela, ce sont les sites Internet eux-mêmes qui érigent des obstacles (évitables) en raison de leur organisation peu conviviale. Afin de mieux étudier les besoins et les exigences de la génération des plus de 65 ans en ce qui concerne la conception conviviale de sites Web, des personnes âgées ont été questionnées sur le sujet « Internet adapté à l'âge » dans le cadre d'un projet de la ZHAW (Haute école spécialisée dans les sciences appliquées) en collaboration avec le Centre de gérontologie de l'Université de Zurich. Pour cela, quatres groupes de discussion ont été formés avec au total 24 participants âgés (moyenne d'âge 73 ans) qui utilisent régulièrement Internet. Le but de ces groupes de discussion était de recueillir le point de vue des utilisateurs âgés ainsi que les barrières créées par la conception du site Internet. Un résultat important a été que les participants accordaient une grande valeur à la bonne présentation des sites Web. C'est ainsi que certains ont rapporté qu'ils n'auraient déjà pas pu utiliser comme ils le souhaitaient des sites Web à cause d'une mauvaise présentation/orientation de l'utilisateur. A la fin du questionnement des groupes, les déclarations ont été comparées à la version actuelle de la littérature spécialisée, discutée et complétée au sein de deux cercles d'experts.

Sur la base de cette collecte de plusieurs niveaux menée à travers l'étude de la littérature, les groupes d'utilisateurs et les cercles d'experts, des recommandations ont pu être formulées pour une « conception des sites Web adaptée à l'âge ». Ces recommandations englobent entre autres les domaines comme la clarté des sites Web, la lisibilité et la perception des contenus ainsi que la convivialité de la navigation.

Ces recommandations ont été systématiquement recueillies dans une brochure. Cette brochure ainsi qu'un module d'e-learning sont disponibles sous www.ageweb.ch. Une deuxième brochure sur le sujet «Applications mobiles adaptées à l'âge » est prévue pour le début 2016.

Auteur : Prof. Dr. Alireza Darvishy est doyen et directeur du département ICT-Accessibility à la Zürcher Hochschule für Angewandte Wissenschaften (zhaw). Contact : alireza.darvishy@zhaw.ch. 